PROCEEDINGS OF THE

AMERICAN MATHEMATICAL SOCIETY

Volume 139, Number 12, December 2011, Pages 4377-4384

S 0002-9939(2011)10853-0

Article electronically published on April 22, 2011

\title{
THE RADON-NIKODYM PROPERTY FOR SOME BANACH ALGEBRAS RELATED TO THE FOURIER ALGEBRA
}

\author{
EDMOND E. GRANIRER
}

(Communicated by Michael T. Lacey)

\begin{abstract}
The Radon-Nikodym property for the Banach algebras $A_{p}^{r}(G)=$ $A_{p} \cap L^{r}(G)$, where $A_{2}(G)$ is the Fourier algebra, is investigated. A complete solution is given for amenable groups $G$ if $1<p<\infty$ and for arbitrary $G$ if $p=2$ and $A_{2}(G)$ has a multiplier bounded approximate identity. The results are new even for $G=\mathbb{R}^{n}$.
\end{abstract}

\section{INTRODUCTION}

The Fourier algebra of the torus $\mathbb{T}$, namely $A(\mathbb{T})$, is in fact $\ell^{1}(\mathbb{Z})$ and as such has the Radon-Nikodym property (RNP), a property possessed by any Banach space which is isomorphic to an $\ell^{1}$ space (see the sequel and $[\mathrm{DiU}]$ ). However, $L^{1}(\mathbb{R})$, hence $A(\mathbb{R})$, does not possess this property.

A Banach space $X$ has the RNP iff its unit ball wants to be weakly compact, but just cannot make it, as beautifully put orally by Jerry Uhl.

In fact $X$ has the RNP iff any norm bounded closed convex subset $Y$ is the norm closed convex hull of its strongly exposed points.

For dual Banach spaces $X$ the RNP is equivalent to any such $Y$ being the norm closed convex hull of its extreme points, i.e., $X$ having the Krein-Milman property (KMP) (see [DiU], p. 128, for at least 17 conditions which are equivalent to the $\mathrm{RNP})$.

In what follows, $G$ will always denote a locally compact group. The results are new even if $G=\mathbb{R}^{n}$.

Clearly $A(G)$ has the RNP if $G$ is compact abelian, and yet $A(G)$ does not have the RNP if $G$ is abelian but noncompact. However, if $K$ is any compact subset of the abelian group $G$, then $A_{K}(G)=\{u \in A(G)$; spt $u \subset K\}$ does have the RNP (here $\operatorname{spt} u=\operatorname{cl}\{x \in G ; u(x) \neq 0\}$ and cl denotes closure). The above results can be proved using the Fourier transform and other tools of abelian harmonic analysis. These are not available anymore if $G$ is not abelian.

For any $G, \forall 1<p<\infty$, let $A_{p}(G)$ denote the Figa-Talamanca-Herz Banach algebra as defined by Herz in [Hz1, thus generated by $L^{p^{\prime}} * L^{p \vee}$; see the sequel. Hence $A_{2}(G)$ is the Fourier algebra of $G$ (i.e., $A(G)$ as described by Eymard in Ey1).

Received by the editors November 15, 2009 and, in revised form, October 14, 2010 and October 20, 2010.

2010 Mathematics Subject Classification. Primary 43A15, 46J10, 43A25, 46B22; Secondary 46J20, 43A30, 43A80, 22E30.

(C)2011 American Mathematical Society Reverts to public domain 28 years from publication 
We have proved in Gr2 that for any $G$ and any compact $K \subset G$ and any $1<p<\infty, A_{K}^{p}=\left\{u \in A_{p}(G)\right.$; spt $\left.u \subset K\right\}$ has the RNP. This seems to be the first paper in which the RNP has been studied for function subalgebras of $A_{p}(G)$.

It is the purpose of this paper to investigate the RNP for the Figa-TalamancaHerz-Lebesgue Banach algebras $A_{p}^{r}(G)=A_{p} \cap L^{r}(G), \forall 1 \leq r \leq \infty$, equipped with the norm

$$
\|u\|_{A_{p}^{r}}=\|u\|_{A_{p}}+\|u\|_{L^{r}}, \quad \text { see Gr1. }
$$

We give a complete solution to the problem in the case when $G$ is unimodular and either is amenable and $1<p<\infty$, or $p=2$ and $A_{2}(G)$ has a multiplier bounded approximate identity.

For abelian $G$, the Banach algebras $A_{2}^{r}(G)=\left\{f \in L^{1}(\hat{G}) ; \hat{f} \in L^{r}(G)\right\}$ have been around for a long time. Their study started in a beautiful paper by Larsen, Liu and Wang [LLW]. The first paper in which $A_{p}^{1}(G)$, for non-abelian $G$ was studied is by Lai and Chen $\mathrm{LCh}$. It was this paper which gave us the impetus to study in Gr1 functional analytic properties of the Banach algebras $A_{p}^{r}(G)$, for arbitrary $G$.

It has been proved by W. Braun in an unpublished manuscript $\mathrm{Br}$ that $A_{p}^{1}(G)$ has the RNP if $G$ is amenable, employing the method used in our paper Gr2].

The main result of this paper is the following:

Theorem 2.2. Let $G$ be unimodular and either

(1) $1<p<\infty$ and $G$ is amenable or

(2) $p=2$ amd $A_{2}(G)$ has a multiplier bounded approximate identity.

Then $\forall 1 \leq r \leq \max \left(p, p^{\prime}\right), A_{p}^{r}(G)$ has the $R N P$.

If $G$ is $\mathrm{SL}(2, \mathbb{R})$ or $\mathrm{SL}(2, \mathbb{C})$, then $A_{2}^{r}(G), \forall 2<r \leq \infty$, does not have the $R N P$. Hence the above is the best one can do.

A crucial step in the proof is the identification of $A_{p}^{r}(G) \forall 1 \leq r \leq \max \left(p, p^{\prime}\right)$ as a dual Banach space, namely:

Theorem 2.1. Let $G$ be unimodular and either (1) $p=2$ or (2) $G$ is amenable or discrete, and $1<p<\infty$. Then $W_{p} \cap L^{r}(G)=A_{p} \cap L^{r}(G)=A_{p}^{r}(G)$ is a dual Banach space, $\forall 1 \leq r \leq \max \left(p, p^{\prime}\right)$.

The above equality fails even if $p=2$ and $G=\mathbb{T}$, and $r>2$.

\section{MAin Results}

In what follows, $A_{p}(G)$ will denote the Figa-Talamanca-Herz Banach algebra as defined in [Hz1]. (This algebra is denoted by $A_{p^{\prime}}(G)$ in Ey2 and in Gr1.)

Hence $u \in A_{p}(G)$ if and only if $u=\sum u_{n} * v_{n}^{\vee}$, where $u_{n} \in L^{p^{\prime}}(G), v_{n} \in$ $L^{p}(G), \sum\|u\|_{L^{p^{\prime}}}\left\|v_{n}\right\|_{L^{p}}<\infty$, the infimum of these being the norm of $u \in A_{p}$, and $p^{-1}+\left(p^{\prime}\right)^{-1}=1,1<p<\infty$.

Let $A_{p}^{r}(G)=A_{p} \cap L^{r}(G)$, with norm given by $\|u\|_{A_{p}^{r}}=\|u\|_{A_{p}}+\|u\|_{L^{r}}$ (see Gr1] for their properties). Let $r^{\prime}$ be given by $r^{-1}+\left(r^{\prime}\right)^{-1}=1$ and $r^{\prime}=\infty$, if $r=1$.

Denote by $P M_{p}(G)=A_{p}(G)^{*}$, the Banach space dual of $A_{p}(G)$, and let $P F_{p}(G)$ denote the norm closure of $L^{1}(G)$ in $P M_{p}(G)$. Let $W_{p}(G)=P F_{p}(G)^{*}$. $W_{p}(G)$ is a subspace of $L^{\infty}(G)$, a Banach algebra under pointwise operations, and an $A_{p}(G)$ module; see Cowling Co2, pp. 91 and 94. 
Proposition 2.1. Let $G$ be a locally compact group. Denote $W_{p}^{r}(G)=W_{p} \cap L^{r}(G)$, with norm given by $\|u\|=\|u\|_{W_{p}}+\|u\|_{L^{r}}$. Then $W_{p}^{r}(G)$ is the dual of a Banach space.

Proof. Denote $X=P F_{p}(G), Y=L^{r^{\prime}}(G)$, with their appropriate norms, and let $Z=X \times Y$, with norm given by $\|(x, y)\|=\max (\|x\|,\|y\|)$. Then $Z^{*}=(X \times Y)^{*}=$ $\left(X^{*} \times Y^{*}\right)=\left(W_{p} \times L^{r}\right)$, with norm $\|(u, f)\|=\|u\|_{W_{p}}+\|f\|_{L^{r}}$. Now consider the diagonal $N=\left\{(w, w) ; w \in W_{p}^{r}\right\}$ as a subspace of $Z^{*} . N$ is isometric to the Banach space $W_{p}^{r}$. The last will be the dual of a Banach space if $N$ is a $w^{*}$ closed subspace of $Z^{*}$. Then $N=\left(Z / N_{0}\right)^{*}$, where $N_{0}$ is the annihilator of $N$ in $Z$; see [Ru2], 4.7-4.9.

Hence let $\left(w_{\alpha}, w_{\alpha}\right) \rightarrow\left(w_{0}, f_{0}\right), w^{*}$ in $Z^{*}$, where $w_{\alpha} \in W_{p}^{r}, w_{0} \in W_{p}, f_{0} \in L^{r}$. Thus for any $\varphi \in P F_{p}, f \in L^{r^{\prime}},\left(w_{\alpha}, \varphi\right)+\left(w_{\alpha}, f\right) \rightarrow\left(w_{0}, \varphi\right)+\left(f_{0}, f\right)$. Now choose $a \in L^{1} \cap L^{r^{\prime}}$. By first choosing $\varphi=a, f=0$ we get that $\left(w_{\alpha}, a\right) \rightarrow\left(w_{0}, a\right)=$ $\int w_{0} a \mathrm{~d} x$. But if we choose $\varphi=0, f=a$, we get that $\left(w_{\alpha}, a\right) \rightarrow\left(f_{0}, a\right)=\int f_{0} a \mathrm{~d} x$. It follows that for all $a \in L^{1} \cap L^{r^{\prime}}, \int\left(w_{0}-f_{0}\right) a \mathrm{~d} x=0$. Since $f_{0}$ has $\sigma$ compact support, it follows that $w_{0}=f_{0}$ a.e. Hence, $w_{0} \in W_{p}^{r}$, and $W_{p}^{r}$ is a dual Banach space.

Let $B_{p}(G)$ denote the pointwise multipliers of $A_{p}(G)$, with the multiplier norm. It is proved by Cowling in Co2, Theorem 5 , that $W_{p}(G) \subset B_{p}(G)$ and that $W_{p}(G)$ is isomorphically isometric to $B_{p}(G)$ if and only if $G$ is amenable.

The next theorem has been proved in the case when $G$ is abelian and $p=2$ by Liu and van Rooij in $\mathrm{LiR}$, Corollary 2.6, using tools involving the Fourier transform. It has been proved in $\mathrm{BrFei}$ ] that $A_{p}^{1}(G)$ is a dual Banach space in the case when $G$ is amenable, as pointed out by Braun in $\mathrm{Br}$. A substantial improvement of these results is the next result, for which the abelian methods fail.

Theorem 2.1. Let $G$ be unimodular and either (1) $p=2$ or (2) $G$ is amenable or discrete, and $1<p<\infty$. Then

$$
W_{p} \cap L^{r}(G)=A_{p} \cap L^{r}(G)=A_{p}^{r}(G), \quad \forall 1 \leq r \leq \max \left(p, p^{\prime}\right) .
$$

Hence $A_{p}^{r}(G)$ is a dual Banach space for all $1 \leq r \leq \max \left(p, p^{\prime}\right)$.

Remark 2.1. This theorem cannot be improved in terms of $p$ and $r$, even for the torus $\mathbb{T}$ and $p=2$.

In fact, as noted in [LiR, p. 40, it has been proved by Hewitt and Zuckerman in [HZ, Corollary 4.5, that if $G$ is the torus or any 0-dimensional compact abelian group with dual group $\Gamma$, there exists a singular probability measure $\mu$ on $G$, such that $\mu * \mu$ is absolutely continuous and such that $\sum|\hat{\mu}(\gamma)|^{2 r}<\infty, \forall r>1$.

Hence, $W_{2} \cap L^{r}(\Gamma) \neq A_{2} \cap L^{r}(\Gamma), \forall r>2$.

Proof. Let $U_{\alpha}$ be a relatively compact base of neighborhoods of $e$ and $V_{\alpha}=V_{\alpha}^{-1}$, and let there be open neighborhoods of $e$ such that $V_{\alpha}^{2} \subset U_{\alpha}$. Denote $s_{\alpha}=$ $\lambda\left(V_{\alpha}\right)^{-1} 1_{V_{\alpha}}$. Let $t_{\alpha}=s_{\alpha} * s_{\alpha}^{\sim}$, where $f^{\sim}(g)=f\left(g^{-1}\right) \Delta\left(g^{-1}\right)$. Let $e_{\alpha}=t_{\alpha} * t_{\alpha}$; thus $e_{\alpha}$ is the square of a special operator, as in [Fe.

We now apply the lemma on p. 129 of $\mathrm{Fe}$ (which is an improvement of our lemma 1 on p. 461 of $\left[\mathrm{GrL}\right.$ ) to $w \in W_{p} \cap L^{p}(G)$ and get that $\left\|e_{\alpha} * w-w\right\|_{W_{p}} \rightarrow 0$. However $e_{\alpha} \in L^{p^{\prime}}$ and thus $e_{\alpha} * w \in L^{p^{\prime}} * L^{p}(G)^{\vee} \subset A_{p}(G)$, since $G$ is unimodular. 
If $p=2$, then the unit ball of $P F_{2}(G)$ is strongly dense in the unit ball of $P M_{2}(G)=A_{2}(G)^{*}$, even if $G$ is not amenable, by Kaplanski's density theorem. If $G$ is amenable and $1<p<\infty$, the same holds true by Theorem 5 of Herz [Hz1]. It follows that the $W_{p}$ norm restricted to $A_{p}(G)$ coincides with the $A_{p}$ norm in both cases.

Now $e_{\alpha} * w \in A_{p}(G)$ is a $W_{p}$, hence an $A_{p}$ norm Cauchy net. It follows that $w \in A_{p}(G)$. Hence $W_{p} \cap L^{p}(G)=A_{p} \cap L^{p}(G)$ in both cases. Now $W_{p}$ contains only bounded functions; thus $W_{p} \cap L^{r} \subset W_{p} \cap L^{p}, \forall 1 \leq r \leq p$. Hence, for such $r$, $W_{p} \cap L^{r} \subset A_{p} \cap L^{p} \cap L^{r}=A_{p} \cap L^{r}=A_{p}^{r}$.

Now $W_{p}(G)^{\vee}=W_{p^{\prime}}(G)$ and $A_{p}(G)^{\vee}=A_{p^{\prime}}(G)$; see Co2, p. 91. Since $G$ is unimodular it is clear that

$$
\left(W_{p} \cap L^{r}\right)^{\vee}=W_{p}^{\vee} \cap L^{r}=W_{p^{\prime}} \cap L^{r}=A_{p^{\prime}}^{r}, \quad \forall 1 \leq r \leq p^{\prime} .
$$

If $p \leq 2$, then $W_{p} \cap L^{r}=\left(W_{p^{\prime}} \cap L^{r}\right)^{\vee}=\left(A_{p^{\prime}}^{r}\right)^{\vee}=A_{p}^{r}, \forall 1 \leq r \leq p^{\prime}$.

If $G$ is discrete, one can omit the amenability of $G$ since then $L^{p^{\prime}} \cup L^{p}=L^{p^{\prime}} *$ $\delta_{e} \cup \delta_{e} * L^{p} \subset A_{p}$. Let $s=\max \left(p, p^{\prime}\right)$. If $1 \leq r \leq s$, then $W_{p} \cap L^{r} \subset W_{p} \cap L^{s} \subset A_{p}$. Hence $W_{p} \cap L^{r} \subset A_{p}^{r}$.

We will prove in what follows that $A_{p}^{r}(G)$, for $1 \leq r \leq \max \left(p, p^{\prime}\right)$, has, as a Banach space, the Radon-Nikodym property (RNP) for certain $G$. This fact has been proved for $A_{p}^{1}(G)$ if $G$ is amenable by W. Braun in an unpublished preprint [Br] using our method in Gr2, where we show that $A_{K}^{p}(G)=\left\{u \in A_{p}\right.$; spt $\left.u \subset K\right\}$, for compact $K$, has the RNP.

Theorem 2.2. Let $G$ be unimodular and either

(1) $1<p<\infty$ and $G$ is amenable or

(2) $p=2$ and $A_{2}(G)$ have a multiplier bounded approximate identity.

Then $\forall 1 \leq r \leq \max \left(p, p^{\prime}\right), A_{p}^{r}(G)$ has the RNP.

Remark 2.2. If $G$ is $\mathrm{SL}(2, \mathbb{R})$ or $\operatorname{SL}(2, \mathbb{C})$, then $A_{2}^{r}(G), \forall 2<r \leq \infty$, does not have the RNP. Yet $A_{2}(G)$ has a multiplier bounded approximate identity (see CaHa, Remark 3.8) and $G$ is unimodular (see HRI (15.30)). Hence the above is the best one can do.

Proof. Assume at first that $G$ is separable metric. Let $K \subset G$ be compact and let $A_{p}^{r}[K]=\left\{u \in A_{p}^{r}(G) ; \operatorname{spt} u \subset K\right\}, A_{p}[K]=\left\{u \in A_{p}(G) ; \operatorname{spt} u \subset K\right\}$. Then the identity $I: A_{p}^{r}[K] \rightarrow A_{p}[K]$ is $1-1$, onto and continuous; hence it is bicontinuous. $A_{p}(G)$ is norm separable; hence so are $A_{p}^{r}[K], A_{p}[K]$. Now let $K_{n}$ be compact and satisfy $K_{n} \subset$ int $K_{n+1}, \bigcup K_{n}=G$. Let $C_{m}=\left\{v_{n}^{m} ; n \geq 1\right\}$ be norm dense in $A_{p}^{r}\left[K_{m}\right]$; then $\bigcup C_{m}$ is norm dense in $A_{p}^{r}(G)$. Since if $G$ is amenable, then $A_{p}(G)$ has a bounded approximate identity, in both cases (1), $(2), A_{p}(G)$ has a multiplier bounded approximate identity. By Gr1], p. $408, A_{p}^{r} \cap C_{c}(G)$ is norm dense in $A_{p}^{r}(G)$. Hence let $v \in A_{p}^{r}(G)$ and $\varepsilon>0$. Let $u \in A_{p}^{r} \cap C_{c}(G)$ be such that $\|v-u\|_{A_{p}^{r}}<\varepsilon$. Then spt $u \subset K_{i}$ for some $i>0$. Let $v_{j}^{i}$ satisfy $\left\|u-v_{j}^{i}\right\|_{A_{p}^{r}}<\varepsilon$. Then $\left\|v-v_{j}^{i}\right\|_{A_{p}^{r}}<2 \varepsilon$.

Now, separable dual Banach spaces have the RNP (see DiU], p. 218); hence $A_{p}^{r}(G)$ has the RNP for $1 \leq r \leq \max \left(p, p^{\prime}\right)$. 
Assume now that $G$ is arbitrary. By [DiU], p. 217, we need only prove that every separable closed subspace $X$ of $A_{p}^{r}(G)$ has the RNP. Hence, let $\left\{u_{k}\right\}$ be norm dense in $X$. Each $u_{k}$ has $\sigma$-compact support; hence there exists a $\sigma$-compact subgroup $H$ such that $u_{k}=0$ off $H$ for all $k$. By [Hz1, p. 106, $A_{p}(H)$ can be identified isometrically with the subalgebra $\left\{u \in A_{p}(G) ; u=0\right.$ off $\left.H\right\}$. It is readily seen now that the same is the case for the algebras $A_{p}^{r}(H), A_{p}^{r}(G)$.

Denote $1_{H}(x)=1,(0)$ if $x \in H,(x \notin H)$. Then by [Hz1], p. 106, the map $u \rightarrow 1_{H} u$, from $A_{p}(G) \rightarrow A_{p}(H)$, is a retract; thus $\left\|1_{H} u\right\|_{A_{p}(H)} \leq\|u\|_{A_{p}(G)}$. It readily follows that if $\left\{u_{\alpha}\right\}$ is a multiplier bounded approximate identity for $A_{p}(G)$, then $\left\{1_{H} u_{\alpha}\right\}$ is such for $A_{p}(H)$. Since $G$ is unimodular, so is $H$.

Thus we can and shall hence assume that $G$ is $\sigma$-compact. We will prove that the above separable subspace $X$ has the RNP.

Let $V_{n}=\left\{x ;\left\|l_{x} u_{k}-u_{k}\right\|_{A_{p}^{r}}+\left\|r_{x} u_{k}-u_{k}\right\|_{A_{p}^{r}}<1 / n, \forall k \leq n\right\}$, where $l_{x} u(y)=$ $u(x y), r_{x} u(y)=u(y x)$. These are open neighborhoods of the identity $e$ of $G$. Hence there exists a compact normal subgroup $N$ such that $N \subset \bigcap\left\{V_{n} ; 1 \leq n<\infty\right\}$ and such that $G / N$ is separable metric; see Lemma 2.1. Thus, by [Hz1], p. 106, Prop. 6, $A_{p}(G / N)$ can be identified isometrically with $\left\{u \in A_{p}(G) ; l_{x} u=r_{x} u=u, \forall x \in N\right\}$. It follows readily that this is also the case for the algebra $A_{p}^{r}(G / N)$ by noting Weil's formula in [RS, Theorem 3.4.6. Hence the Banach space $X$ is a subspace of the Banach algebra $A_{p}^{r}(G / N)$. Since subspaces of RNP spaces have the RNP (see [DiU], p. 217), it is enough to prove that $A_{p}^{r}(G / N)$ has the RNP.

That $G / N$ is unimodular can be directly proved; see Ga], Theorem V.3.15 and the remarks on p. 267. We will prove that $A_{p}(G / N)$ has a multiplier bounded approximate identity and then use the first part above to finish the proof.

By Hz1, Prop. 6 on p. 106, $A_{p}(G / N)$ can and shall be identified with the subalgebra of $A_{p}(G)$, given by $B=\left\{u \in A_{p}(G) ; \ell_{x} u=r_{x} u, \forall x \in N\right\}$, where $\ell_{x} u(y)=u(x y), r_{x} u=u(y x)$. The onto map $M_{N} u(x)=\int u(x y) \mathrm{d} \lambda_{N}(y)$, where $\lambda_{N}$ is the Haar measure of $N$, from $A_{p}(G) \rightarrow A_{p}(G / N)$ is a Banach space retract; i.e., $\left\|M_{N} u\right\|_{A_{p}(G / N)} \leq\|u\|_{A_{p}(G)}, \forall u \in A_{p}(G)$. Moreover $M_{N}$ is the identity on $A_{p}(G / N)$. It can be directly shown that $M_{N}(u v)=u M_{N}(v), \forall u \in A_{p}(G / N)$, $v \in A_{p}(G)$.

Now let $\left\{u_{\alpha}\right\}$ be a multiplier bounded approximate identity in $A_{p}(G)$. We claim that $\left\{M_{N} u_{\alpha}\right\}$ is such in $A_{p}(G / N)$, since, if $v \in A_{p}(G / N)$, then

$$
\left\|\left(M_{N} u_{\alpha}\right) v-v\right\|_{A_{p}(G / N)}=\left\|M_{N}\left(u_{\alpha} v\right)-M_{N} v\right\|_{A_{p}(G / N)} \leq\left\|u_{\alpha} v-v\right\|_{A_{p}(G)} \rightarrow 0 .
$$

Also,

$$
\left\|\left(M_{N} u_{\alpha}\right) v\right\|_{A_{p}(G / N)}=\left\|M_{N}\left(u_{\alpha} v\right)\right\|_{A_{p}(G / N)} \leq\left\|u_{\alpha} v\right\|_{A_{p}(G)} \leq K\|v\|_{A_{p}(G)},
$$

for some $K>0$, since $\left\{u_{\alpha}\right\}$ is a multiplier bounded approximate identity in $A_{p}(G)$.

To complete the above proof we still need to prove the following lemma, which is an improvement of Theorem 8.7 in [HRI], where it is assumed that $G$ is compactly generated. The proof is inspired by Prop. 6 in [Hz1. Lemma 2.1 gives, in addition, a new proof for [HRI] (8.7).

Lemma 2.1. Let $G$ be a $\sigma$-compact locally compact group and let $U_{n}, n=1,2, \ldots$, be neighborhoods of the identity. Then there exists a compact normal subgroup $N \subset \bigcap\left\{U_{n} ; 1 \leq n<\infty\right\}$, such that $G / N$ is separable metric. 
Proof. Let $V_{1}=V_{1}^{-1}$ be a relatively compact neighborhood of $e$ such that $V_{1}^{4} \subset U_{1}$. If $V_{1}, \ldots, V_{n-1}$ were chosen, choose the neighborhood $V_{n}=V_{n}^{-1}$ of $e$ such that $V_{n}^{4} \subset\left\{\cap U_{k}, 1 \leq k \leq n\right\} \cap V_{n-1}$. Let $f_{n} \in C_{c}(G)$ be such that $0 \leq f_{n} \leq 1, f_{n}=1$, on $V_{n}$ and $f_{n}=0$ off $V_{n}^{2}$. Denote $v_{n}(x)=\left\|\lambda(x) f_{n}-f_{n}\right\|_{\infty}$, where $\lambda(x) f(y)=$ $f\left(x^{-1} y\right)$. Then, in the language of Hz1, p. 107, $v_{n}$ is a continuous invariant pseudometric on $G$; i.e., it satisfies $0 \leq v_{n}<\infty, v_{n}(e)=0, v_{n}(x)=v_{n}\left(x^{-1}\right)$ and $v_{n}(x y) \leq v_{n}(x)+v_{n}(y)$, for all $x, y$.

We note that $\lambda(x) f_{n}$ and $f_{n}$ have disjoint supports if $x \notin V_{n}^{4}$; thus $v_{n}(x)=\left\|f_{n}\right\|$ for such $x$. Hence the set $\left\{x ; v_{n}(x)=0\right\}$ is a compact, not necessarily normal, subgroup $M_{n}$ of $G$. Now let $\omega(x)=\sum_{n} 2^{-n}\left[v_{n}(x)\left(1+v_{n}(x)\right)^{-1}\right]$. Then $\omega(x)$ is again a continuous invariant pseudometric on $G$ (since $g(t)=t /(1+t)$ is increasing on $[0, \infty))$, and $\omega(x)=0$, iff $v_{n}(x)=0, \forall n$, iff $x \in M_{n} \subset V_{n}^{4} \subset U_{n}, \forall n$. Hence $\omega^{-1}(0)=M \subset \bigcap U_{n}$, where $M=\bigcap M_{n}$, is a compact, not necessarily normal, subgroup of $G$.

Now let $G=\bigcup K_{n}$, where $K_{n}$ are compact sets such that $e \in K_{n} \subset K_{n+1}$. For each $n$ let $\omega_{n}(x)=\sup \left\{\omega\left(y x y^{-1}\right) ; y \in K_{n}\right\}$, again a continuous invariant pseudometric on $G$. Let $\tau=\sum 2^{-n} \omega_{n}\left(1+\omega_{n}\right)^{-n}$. Then $\tau$ is a continuous invariant pseudometric such that $\tau^{-1}(0)=N$ is a compact normal subgroup such that $N \subset$ $M \subset \bigcap U_{n}$ and $d(x, y)=\tau\left(x^{-1} y\right)$ is a continuous (even left invariant) pseudometric (as in [HRI]) on $G$ which induces a metric on $G / N$ and which renders $G / N$ with the quotient topology into a separable metric space.

Clearly $\tau(x) \geq \omega_{1}(x) / 4, \forall x \in G$, since $0 \leq \omega(x) \leq 1$. If $\tau(x)=0$, then $\omega(x)=0$, since $e \in K_{1}$; hence $N \subset M$. In addition, if $a \in G$, then $a \in K_{k}$ for some $k$ and $0=\omega_{k}(x) \geq \omega\left(a x a^{-1}\right)$. Thus $\omega\left(y x y^{-1}\right)=0, \forall y \in G$. But then $\omega_{n}\left(a x a^{-1}\right)=\sup \left\{\omega\left(y a x a^{-1} y^{-1}\right) ; y \in K_{n}\right\}=0$, for all $n$. Hence $\tau\left(a x a^{-1}\right)=0$ and $N=\tau^{-1}(0)$ is a compact normal subgroup.

Now let $d(x, y)=\tau\left(x^{-1} y\right)$ for $x, y$ in $G$. Then $d$ is a continuous left invariant pseudo-metric, as in [HRI], (8.1); i.e., $d(x, x)=0, d(x, y)=d(y, x)$, and

$$
0 \leq d(x, y) \leq d(x, z)+d(z, y), d(x, y)=d(a x, a y) \quad \forall x, y, z .
$$

We note that $\tau\left(x u x^{-1}\right)=0, \forall u \in N, x \in G$; thus $d(x, u x)=0$, for such $u, x$. Hence $d(u x, v y) \leq d(x, y)+d\left(y, u^{-1} v y\right)=d(x, y), \forall u, v \in N, x, y \in G$. But then $d(x, y)=d\left(u^{-1} u x, v^{-1} v y\right) \leq d(u x, v y)$, for such $x, y, u, v$. Thus $D(x N, y N)=$ $d(x, y)$ is a left invariant continuous metric on $G / N$, which clearly induces the quotient topology on $G / N$, since it does so on each compact subset. It is readily seen that $G / N$ is second countable; thus it is separable metric.

Proof of Remark 2.2, If $G=\mathrm{SL}(2, \mathbb{R})$ or $\mathrm{SL}(2, \mathbb{C})$, then $A_{2}^{r}(G)=A_{2}(G), \forall r>2$, by Kunze and Stein [KuS] and by Lipsman [Li], respectively; see also $\mathrm{Co1}$.

We will prove hence that $A_{2}(G)$ does not have the RNP if $G$ is any of the above groups. By Keith Taylor's beautiful result in Tay, Theorem 4.1, we only need to prove that the regular representation is not a direct sum of irreducible representations for the above groups $G$.

Assume the contrary. Then the Plancherel measure $\mu$, which is unique once the Haar measure has been fixed by [Dix], (18.8.2), is necessarily an atomic measure; see Dix (18.8.5). However, this is not the case for the above $G$, as can be seen from Knapp [Kn, II.7, formulas (2.24) and (2.25) on p. 42. In fact one can get from there, by taking $h=f * f^{*}$, where $f$ is a compactly supported smooth function on 
$G$, that $\|f\|_{L^{2}}^{2}=\int_{G^{\wedge}} \operatorname{Tr} \pi(f) \pi(f) * d \mu(\pi)$ where the measure $\mu$ in $\underline{\mathrm{Kn}}$, II.7, (2.24) and (2.25) is not atomic. There are some atoms in (2.25), i.e., for $\operatorname{SL}(2, \mathbb{R})$, which are the "discrete series", but the atoms do not make up everything in the support of $\mu$.

Remark 2.3. The group $\operatorname{SL}(2, \mathbb{R}) \propto \mathbb{R}^{n}, \forall n \geq 2$, does not satisfy condition (2) of Theorem 2.2, see [Do. Yet there are a multitude of non-amenable groups which do satisfy this condition; see $\mathrm{CaHa}$.

\section{ACKNOWLEDGEMENTS}

We acknowledge with thanks correspondence with Michael Leinert and Keith Taylor.

\section{REFERENCES}

[Br] W. Braun: Einige Bemerkungen $Z u S_{0}(G)$ und $A_{p}(G) \cap L^{1}(G)$. Preprint (1983).

[BrFei] W. Braun and Hans G. Feichtinger: Banach spaces of distributions having two module structures. J. Funct. Analysis 51 (1983), 174-212. MR701055 (84h:46062)

[CaHa] J. De Carrière and U. Haagerup: Multipliers of the Fourier algebra of some simple Lie groups and their discrete subgroups. Amer. J. Math. 107 (1985), 455-500. MR784292 (86m:43002)

[Co1] Michael Cowling: The Kunze-Stein phenomenon. Ann. of Math. 107 (1978), 209-234. MR0507240 (58:22398)

[Co2] Michael Cowling: An application of Littlewood-Paley theory in harmonic analysis. Math. Ann. 241 (1979), 83-96. MR531153 (81f:43003)

[DiU] J. Diestel and J.J. Uhl: Vector Measures. Math. Surveys, No. 15, Amer. Math. Soc., 1977. MR.0453964(56:12216)

[Dix] J. Dixmier: Les $C^{*}$ algebres et leur representations. Gauthier-Villars, 1969. MR.0246136 (39:7442)

[Do] B. Dorofaeff: The Fourier algebra of $\mathrm{SL}(2, \mathbb{R}) \propto R^{n}, n \geq 2$ has no multiplier bounded approximate unit. Math. Ann. 297 (1993), 707-724. MR.1245415 (94k:43005)

[DS] N. Dunford and J. Schwartz: Linear Operators. Part 1. General Theory. Interscience Publishers, New York, 1958. MR0117523 (22:8302)

[Ey1] P. Eymard: L'algebre de Fourier d'un groupe localement compacte. Bull. Soc. Math. France 92 (1964), 181-236. MR0228628 (37:4208)

[Ey2] P. Eymard: Algebre $A_{p}$ et convoluteurs de $L_{p}$. Lecture Notes in Math., No. 180, Springer, 1971, 364-381.

[Fe] Gero Fendler: An $L^{p}$-version of a theorem of D.A. Raikov. Ann. Inst. Fourier, Grenoble 35 (1985), 125-135. MR781782 (86h:43003)

[FT] A. Figa-Talamanca: Translation invariant operators on $L^{p}$. Duke Math. J. 32 (1965), 495-501. MR0181869 (31:6095)

[FTP] A. Figa-Talamanca and M. Picardello: Multiplicateurs de $A(G)$ qui ne sont pas dans $B(G)$. C.R. Acad. Sci. Paris 277 (1973), 117-119. MR0333597 (48:11922)

[Ga] Steven A. Gaal: Linear Analysis and Representation Theory. Springer Verlag, New York, Heidelberg, Berlin, 1973. MR0447465 (56:5777)

[GMc] C.C. Graham and O.C. McGehee: Essays in Commutative Harmonic Analysis. Springer Verlag, 1979. MR 550606 (81d:43001)

[Gr1] Edmond E. Granirer: The Figa-Talamanca-Herz-Lebesgue Banach algebras $A_{p}^{r}(G)=$ $A_{p}(G) \cap L^{r}(G)$. Math. Proc. Camb. Phil. Soc. 140 (2006), 401-416. MR2225639 (2007f:46049)

[Gr2] Edmond E. Granirer: An application of the Radon Nikodym property in harmonic analysis. Bull. U.M.I. (5) 18-B (1981), 663-671. MR629430 (83b:43004)

[Gr3] Edmond E. Granirer: Strong and extremely strong Ditkin sets for the Banach algebras $A_{p}^{r}(G)=A_{p}(G) \cap L^{r}(G)$. Canad. J. Math. 63 (2011). 
[GrL] E. E. Granirer and M. Leinert: On some topologies which coincide on the unit sphere of the Fourier-Stieltjes algebra $B(G)$ and the measure algebra $M(G)$. Rocky Mountain J. Math. 11 (1981), 459-472. MR.0722579 (85f:43009)

[HRI] E. Hewitt and K.A. Ross: Abstract Harmonic Analysis, Vols. 1 and 2. Springer Verlag, 1963, 1970. MR0156915(28:158), MR0262773(41:7378)

[HZ] Edwin Hewitt and Herbert Zuckerman: Singular measures with absolutely continuous convolution squares. Proc. Camb. Phil. Soc. 62 (1966), 399-420. MR0193435 (33:1655)

[Hz1] C. Herz: Harmonic synthesis for subgroups. Ann. Inst. Fourier, Grenoble 23 (1973), 91123. MR:0355482 (50:7956)

[Hz2] C. Herz: The theory of $p$ spaces with an application to convolution operators. Trans. Amer. Math. Soc. 154 (1971), 69-82. MR0272952 (42:7833)

[Hz3] C. Herz: On the asymmetry of norms of convolution operators. I.J. Functional Analysis 23 (1976), 11-22. MR0420138 (54:8153)

[Kn] Anthony W. Knapp: Representation Theory for Semisimple Groups. Princeton University Press, Princeton, New Jersey, 1986. MR855239 (87j:22022)

[KuS] R.A. Kunze and E.M. Stein: Uniformly bounded representations and harmonic analysis on the $2 \times 2$ unimodular group. Amer. J. Math. 82 (1960), 47-66. MR0163988 (29:1287)

[LCh] Hang-Chin Lai and Ing-Sheun Chen: Harmonic analysis on the Fourier algebras $A_{1, p}(G)$. J. Austral. Math. Soc. (Series A) 30 (1981), 438-452. MR621559 (82k:43002)

[Li] R.L. Lipsman: Harmonic analysis on SL(n,C). J. Funct. Anal. 3 (1969), 126-155. MR0237716 (38:5997)

[LiR] Teng-sun Liu and Arnoud van Rooij: Sums and intersections of normed linear spaces. Math. Nachrichten. 42 (1969), 29-42. MR0273370 (42:8249)

[LLW] Ron Larsen, Teng-sun Liu and Ju-kwei Wang: On functions with Fourier transforms in Lp. Mich. Math. J. 11 (1964), 369-378. MR0170173 (30:412)

[Lu] Francoise Lust-Piquard: Means on $C V_{p}(G)$-subspaces of $C V_{p}(G)$ with RNP and Schur property. Ann. Inst. Fourier, Grenoble 39 (1989), 969-1006. MR1036340 (91d:43002)

[RS] H. Reiter and J.T. Stegeman: Classical harmonic analysis and locally compact groups. London Math. Soc. Monographs, New Series, 22, Oxford Sci. Publ., 2000. MR1802924 (2002d:43005)

[Ri] N.W. Rickert: Convolutions of $L_{p}$ functions. Proc. Amer. Math. Soc. 18 (1967), 762-763. MR0216301 (35:7136)

[Ru1] W. Rudin: Fourier analysis on groups. Interscience Publ., 1962. MR0152834 (27:2808)

[Ru2] W. Rudin: Functional analysis. McGraw-Hill, 1973. MR0365062 (51:1315)

[Tay] Keith F. Taylor: Geometry of the Fourier algebras and locally compact groups with atomic unitary representations. Math. Ann. 262 (1983), 183-190. MR690194 (84h:43020)

Department of Mathematics, University of British Columbia, Vancouver, BC, Canada V6T $1 \mathrm{Z} 4$

E-mail address: granirer@math.ubc.ca 